\title{
To Analyse the Effect of Relaxation Type on Magnetic Resonance Image Compression Using Compressive Sensing
}

\author{
https://doi.org/10.3991/ijoe.v17i04.20759 \\ Vivek Upadhyaya ${ }^{(\bowtie)}$, Mohammad Salim \\ Malaviya National Institute of Technology, Jaipur, India \\ 2018rec9028@mit.ac.in
}

\begin{abstract}
Medical Imaging and scanning technologies are used to provide better resolution of body and tissues. To achieve a better-quality Magnetic Resonance (MR) image with a minimum duration of processing time is a tedious task. So, our purpose in this paper is to find out a solution that can minimize the reconstruction time of an MRI signal. Compressive sensing can be used to accelerate Magnetic Resonance Image (MRI) acquisition by acquiring fewer data through the under-sampling of $\mathrm{k}$-space, so it can be used to minimize the time. But according to the relaxation time, we can further classify the MRI signal into T1, T2, and Proton Density (PD) weighted images. These weighted images represent different signal intensities for different types of tissues and body parts. It also affects the reconstruction process conducted by using the Compressive Sensing Approach. This study is based on finding out the effect of T1, T2, and Proton Density (PD) weighted images on the reconstruction process as well as various image quality parameters like MSE, PSNR, \& SSIM also calculated to analyze this effect. Meanwhile, we can analyze how many samples are enough to reconstruct the MR image so the problem associated with time and scanning speed can be reduced up to an extent. In this paper, we got the Structural Similarity Index Measure (SSIM) value up to 0.89 \& PSNR value $37.83451 \mathrm{~dB}$ at an $85 \%$ compression ratio for the $\mathrm{T} 2$ weighted image
\end{abstract}

Keywords - Compressive Sensing (CS), Magnetic Resonance Imaging (MRI), Mean Square Error (MSE), Peak Signal to Noise Ratio (PSNR), Structural Similarity Index (SSIM)

\section{$1 \quad$ Introduction}

First of all, we have to focus on the MRI and its system components. So, we can easily differentiate different types of MRI Images and can easily understand the process of Magnetic Resonance Imaging.

\subsection{Magnetic resonance system components}

MRI system is the combination of three major components; a set of main magnetic 
coils, three gradient coils, and an integral RF transmitter coil as shown in Figure 1. A combination of different types of magnetic fields generated by these system components is applied to the patient. The system then reproduces a spatially encoded magnetic resonance signal which will then generate Magnetic Resonance Images. The patient is placed for imaging within the central bore of the magnet as shown in Figure 2. The strength of the magnetic field is represented by Bo; the unit of this Bo is Tesla (T). If we talk about the system specification then we have the MRI system with strength from 0.2 Tesla to 3.0 Tesla. A specified co-ordinate system of three orthogonal axes $\mathrm{x}, \mathrm{y}$, and $\mathrm{z}$ are used to define the direction of the magnetic field. The direction of $\mathrm{z}$ axes is considered to the parallel to Bo.

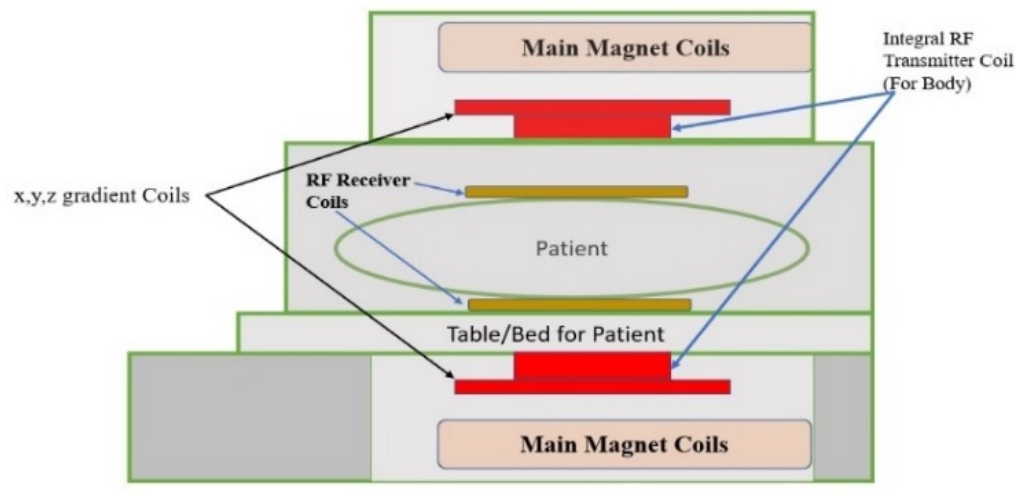

Fig. 1. Diagram representing the relative position of RF receiver coil, RF transmitter Body coil and $\mathrm{x}, \mathrm{y}, \mathrm{z}$ position of the gradient coil
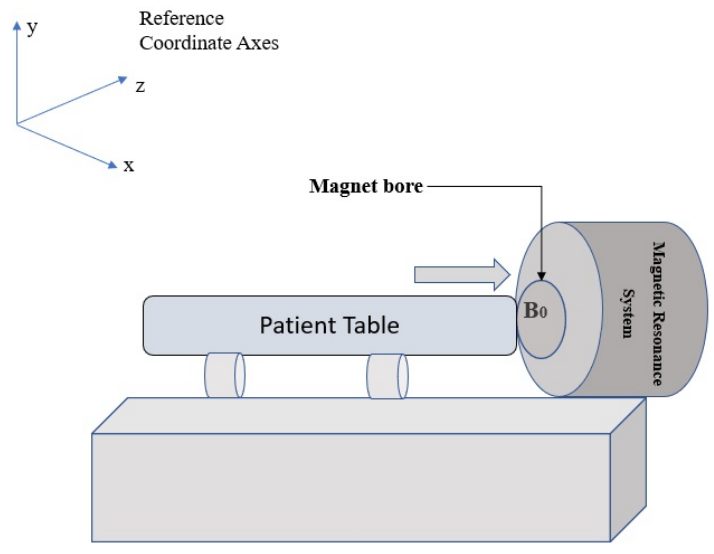

Fig. 2. Cylindrical Bore arrangement for MRI image and reference co-ordinates 


\subsection{Origin of magnetic resonance signals}

The main origin of Magnetic Resonance Signals is from the water or fat of the tissue. Or we can say much precisely that hydrogen nuclei within the water and lipid within the patient or more specifically it is from the hydrogen nuclei (consists of a single proton) contained by water or by lipid molecules. Hydrogen is so much favorable for imaging purposes because of its high sensitivity. Hydrogen Nuclei have a property of nuclear spin which tends to generate a magnetic field by each proton known as the magnetic moment. Normally if we talk about the magnetic moments then they are random but when we apply any external field Bo then it will align towards or opposite to the field. After some time, an equilibrium state is achieved in which the excess spin will further align with the field because the applied field decides the much favorable direction. An excessive amount of magnetic moment from protons will generate a net magnetic field also considered as magnetization (M). This magnetization is aligned with the positive z-axis or we can say with Bo.

To generate an MRI, signal the overall energy which is formed by Net Magnetization, RF field generated by a Radio Frequency (RF) transmitter coil has to be delivered to Protons. This field is applied to Larmor Frequency and is indicated by wo and determined by the equation given below. Now to generate a Magnetic Resonance signal from net magnetization, first of all, a Radio Frequent (RF) magnetic field is generated by using the RF transmitting coil, and then this energy is delivered to the populated protons. Then this overall field is then applied to a frequency which is known as Larmor Frequency and denoted by wo. The equation which is used to determine the Larmor Frequency is given below [1].

$$
w_{0}=\gamma B_{0}
$$

$\gamma$ is a constant known as gyromagnetic ratio \& has a typical value of $42.6 \mathrm{MHz} / \mathrm{Tesla}$ for a proton. We can easily conclude from the equation that the Larmor frequency is highly dependent on the magnetic field density and for the typical value of 1.5 Tesla the Larmor frequency is about $64 \mathrm{MHz}$.

\subsection{Signal characteristics T1, T2 and Proton Density (PD) weighted images}

As per the literature after the immediate action of RF pulse, the spin system comes to its equilibrium position this process is known as relaxation [1]. There are two components of this relaxation process one is longitudinal in the ' $\mathrm{z}$ ' direction and another one is transverse means the ' $\mathrm{XY}$ ' component. The very first relaxation is known as T1 relaxation which is responsible for the recovery of the $\mathrm{z}$ component. Another relaxation process is transverse and due to this, there is some decay in the XY component as it rotates around the z-axis. Both relaxation processes occur at the same time although the transverse relaxation process is much faster for the human tissue. The T1 relaxation process is exponential with the time constant $\mathrm{T} 1$ as mentioned in Figure 3.

Another transverse relaxation is the net combination of magnetic moments of the overall population of protons. Just after the RF pulse, they rotate together coherently so 
that as per the rotation they continuously point in a similar direction as each other within the XY plane. The overall sum of the magnetic moments is thus reduced, as it is the result of a reduction in the transverse field. Then the signal is received by receiver coils it detects that the oscillating field is decaying gradually (known as Free Induction Delay FID). There are two prime causes of this decay first one is based on the interaction between the neighboring protons which create a loss phase coherence known as T2 relaxation. The combined effect of $\mathrm{T} 2$ relaxation and the non-uniformities of the magnetic field is known as T2* relaxation and mentioned in Figure 4.

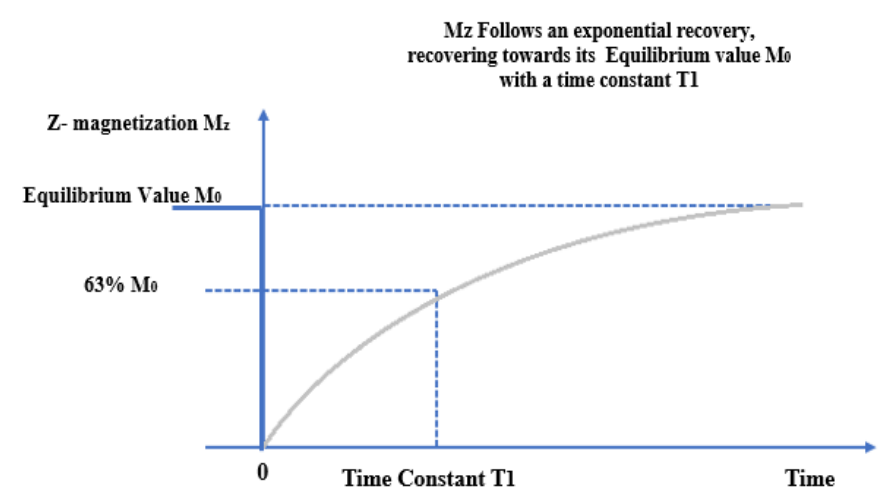

Fig. 3. T1 Relaxation Process

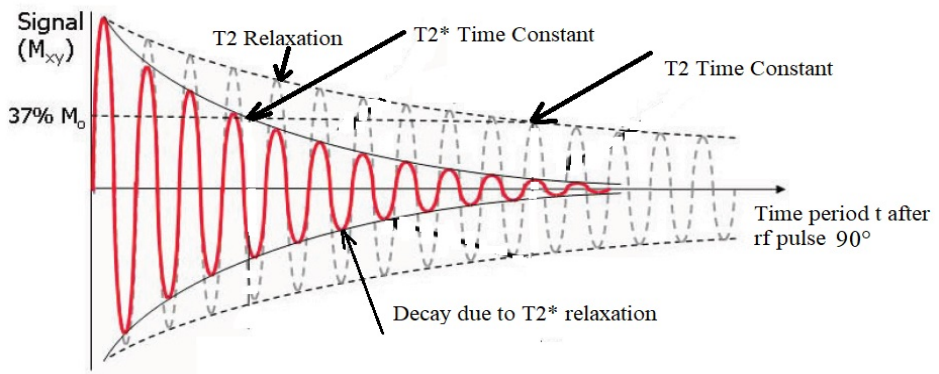

Fig. 4. Transverse $\mathrm{T} 2$ and $\mathrm{T} 2 *$ Relaxation Process

When a Magnetic Resonance Image is set to produce a Proton Density (PD) weighted image, it is the tissues with a higher concentration or density of protons (hydrogen atoms). This produces a very strong signal and appears the brightest part of the image. A very simple way to find out the Proton Density weighted images is to compare the fluid against the fat signal. The fluids normally appear greyish white and have a very similar appearance as the fat in the body. In next section we are moving towards Compressive Sensing theory. 


\subsection{Introduction to compressive sensing theory}

Nyquist, Shannon explored the theoretically based phenomena of this revolution and Whittaker's research on continuous-time band-related signals. Their results exhibit the different types of signals like audio, image, video signal that must be needed at the Nyquist rate for data reconstruction from the equally separate samples. The Nyquist rate of the desired signal should be double the largest frequency. The Digitalized signal has the best sensing and processing quality. The Digital domain-based system is more powerful, flexible, consequently, cheaper and more widely used than other analog counterparts. This work is much appreciated in many exercises but the Nyquist is too high it is more difficult to handle the whole samples. Hence it is too costly and impossible to design a device that can tackle the sample at the Nyquist rate that's would be necessary [2]. Thus, we have challenging groundwork required to create a powerful computational and processing system in the communication field, medical imaging, radar, remote surveillance, video, spectroscopy, data analysis, and much more application [3].

As we know that compression of a signal or mainly, we can say that compressive sensing now a day is a topic on which very much research is going on, but this is also true that some work or various ideas also produced related to this in the $18^{\text {th }}$ century too. In 1795 an algorithm is introduced by proxy for calculation of parameters which are connected to a small number of complicated exponentials, those samples with the noisy [4]. At the opening season of the 1900s an advanced theory, when Caratheodory presents a positive all $\mathrm{k}$ linear combination of sinusoids whose values can be evaluated by $\mathrm{t}=0$ and at any other $2 \mathrm{k}$ point in the time domain. [5][6]. When the value of $\mathrm{k}$ is too small and the assumed value of frequency is high then the representation is apart from the small set of measurements then the sample is below the Nyquist rate.

Compressive sensing is used in many applications with an effective result. Medical imaging is the most important area where CS is used to safely store the diagnostic quality where it accesses and increases the speed because of some factor in the pediatric MRI [7]. Some broad applications provoke the research program of CS thru the introduced some practical proposal and implement many other applications for numerical application and sub-Nyquist sampling systems [8, 9, 10], compressive imaging architectures, and compressive sensor networks [11].

The main objective of compressive sensing is to give up to date investigation and easy concept of some effective results. We get many aspects and result regarding CS after the investigation of every different report. It's depending on the basic starting of the CS.

\section{$2 \quad$ Literature Review}

In the previous section, we have already studied the MRI concepts and the introduction to compressive sensing theory. Now in this section, we are going to elaborate on some work that is done by other researchers in this domain.

Morteza Mardani et al. discussed a new approach "Deep Generative Adversarial Neural Networks for Compressive Sensing (GANCS)" for the compression of MRI 
signals [12]. In this approach, they use under-sampled data for the reconstruction of high-quality MRI signals. These reconstructed images are consistent with the real MRI data.

As per the analysis done nowadays, it is very much clear that $\mathrm{L}_{1}$ regularization suffered from bias problems which will further overcome by using $\mathrm{L}_{1 / 2}$ regularization [13]. Two algorithms for the reconstruction are described in this paper by the author, one is smoothing IHTA and another is projected IHTA. IHTA is referred to as the iterative half thresholding algorithm. The author uses three different popular masking algorithms for experimental purposes and uses the real Magnetic Resonance Image data.

In this paper author explained a novel approach for the MRI compression technique which is the "Learning-Based Compressive MRI". Both noisy and noiseless compression methods are used by the authors [14]. This learning algorithm has access to a set of data and then does the compression according to a specified algorithm which provides a better compression and reconstruction level. The numerical evidence is also given in the paper in the form of PSNR and SSIM values.

Yan $\mathrm{Xi}$ et al. presented a simultaneous CT-MRI image reconstruction method with compressive sensing (CS) and Structural Coupling (SC) as a key technique [15]. The process which is specified by the authors can combine with two or more tomographic imaging modalities. The process is time-effective, cost-effective and so much effective for image performance and biomedical imaging applications.

A local low-rank regularization framework is proposed by the authors in this paper for MRI signal reconstruction. LLR-IRPA and CLEAR algorithms are used for the reconstruction purpose and the results for the same are also discussed in the paper [16]. The paper describes that the reconstruction depends on the sampling pattern which is used for this purpose. So, this LLR- IRPA implicates that we can easily use LLR- based regularization in Medical Imaging applications.

A multi-level compression method is discussed by Tariq Tashan et al. in this paper. The method is applied to the MRI signal and then the peak signal to noise ratio calculated for six samples of MRI images. In this paper, the selection of the compression level is based on SI (sparsity index), and then according to this algorithm compression and reconstruction take place. The given results have shown $7.03 \mathrm{~dB}$ in PSNR and $23.76 \%$ improvement in the compression level to the traditional algorithm [17].

So, these are some points which are discussed by some researchers in their research. Now we are moving towards some mathematical background of the compressive sensing which is considered by us in this paper.

\section{Mathematical Modelling Behind CS-MRI}

According to the Compressive sensing theory, the compressible signal should be sparse in any mathematical domain then only we can reconstruct the original signal by using few samples. The same approach is applied to the MRI signals, here we consider only the samples which have a large value of coefficients and discard other small value coefficients. Other important mathematics is given in the next subsection. 


\subsection{Sparse representation of signal}

A is a real, finite length, discrete-time signal. To represent the input signal in the form of a vector in $\mathrm{R}^{\mathrm{N}}$ vector space as the $\mathrm{Nx} 1$ column vectors like $\mathrm{A}$ [0], $\mathrm{A}[1], \ldots \ldots . \mathrm{A}[\mathrm{N}]$. This vector property used by Basis vector in which any high dimension signal in vector space $\mathrm{R}^{\mathrm{N}}$ can be represented in terms of basis vectors $\left\{\Psi_{j}\right\}_{j=1}^{N} \cdot\left\{\Psi_{h}\right\}_{h=1}^{N}$ is the columns of complete basis matrix $\Psi$ of order $\mathrm{NxN}$.

$$
A=\sum_{j=1}^{N} S_{j} \Psi_{j}
$$

or

$$
A=\Psi S
$$

Here $S_{j}, \mathrm{j}=1,2 \ldots . \mathrm{N}$ are the column component of the $\mathrm{S}$ matrix of order Nx1. $S_{j}$ can be computed by

$$
S=\left\langle A, \Psi_{j}\right\rangle=\Psi^{T} A
$$

Signal $A$ is a representation in the time or space domain while the signal is presented in the form of the basis matrix $\psi$ domain by $S$ for sparse representation of signal A. For the proper representation of the sparse signal, it is required that it hold some $(\mathrm{K})$ large magnitude components and discarded (N-K) small magnitude coefficients. Most of the energy and important information of the signal is condensed in the large magnitude coefficients (which represent K-sparse) which are used to recover the original structure of the signal. These K-sparse signals are encoded for transmission.

\subsection{Measurement matrix}

As mentioned above all $\mathrm{N}$ components must be calculated only $\mathrm{K}(\mathrm{K}<<\mathrm{N})$ which are required, Hence the cost gradually increases. The process which includes the inner product of the input signal \& vector set $\left\{\phi_{j}\right\}_{j=1}^{M}$ is the measurement process that gives.

$$
B_{h}=\left\langle A, \phi_{h}\right\rangle-\phi_{h}^{T} A
$$

Where $\mathrm{B}_{\mathrm{h}}$ is the column component of the matrix $B$ of the order Mx1 and $\phi_{h}^{T}$ are the rows element of $\phi$ a matrix of order $\mathrm{MxN}$.

$$
B=\Phi A=\Phi \Psi S=E S
$$

Where $\mathrm{E}=\Phi . \Psi$ is an $\mathrm{M} \times \mathrm{N}$ matrix. 
$\Phi$ is definite and stable independent from the input of $\mathrm{X}$ then there is some designing problem to make a stable $\Phi$ matrix, in which when we design a basis matrix it needs to sure that the elements of information of signal should not be disturbed, second thing is that the measurements $\mathrm{M}$ is equal to the number of sparse $\mathrm{K}$ while recovering the signal.

As we know that we have to consider under-sampled values for the compressive sensing approach that's why the flow diagram for the process is given below which represents how a magnitude MR image is treated under the compressive sensing methodology.

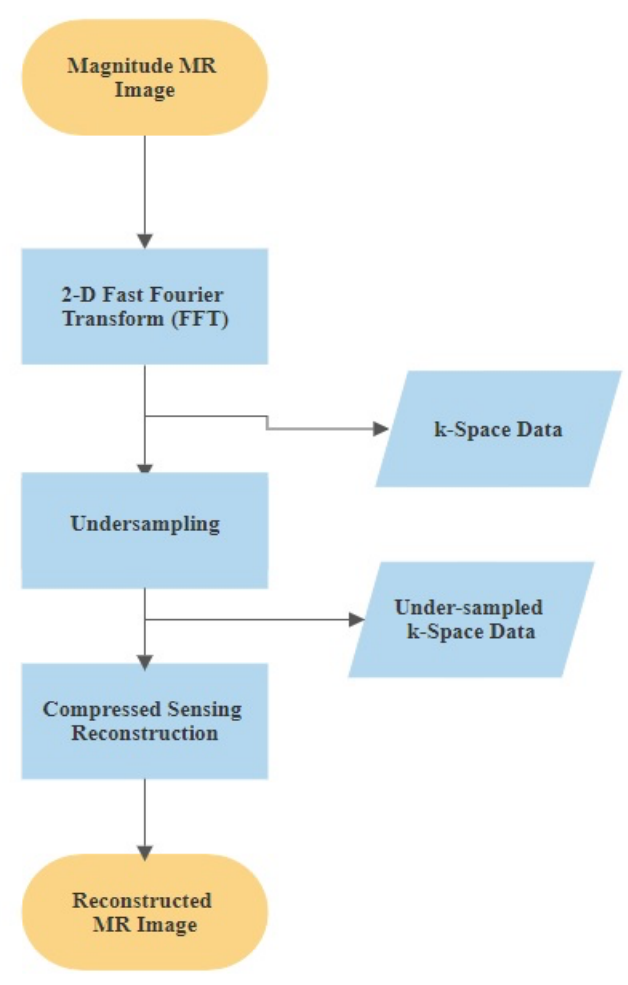

Fig. 5. Flow Diagram for the reconstruction of MR image using magnitude signal

Improvement in the reconstructed MR image quality index parameters (PSNR \& SSIM) is our prime concern in this paper. First, we decide the user-defined value of the compression level, as we have a total number of samples in the image so we can easily able to define the value of the compression level. Now here is the case after defining the value of the compression level we have to design the basis and sensing matrices according to the total number of samples in the image. $1_{1}$ reconstruction algorithm is used for the recovery of MRI images. DCT and Gaussian random matrices are used as the Basis and Sensing matrix at the encoder side. An algorithm consists of these steps given below: 
- Step1: MRI image as an Input (x)

- Step2: Convert whole image in to number of discrete samples $(\mathrm{N})$

- Step3: Calculate Sparsity of Image (S)

- Step4: Decide the Number of Samples required (M) for reconstruction (can be changed)

- Step5: Generate Basis ( $\Psi=\mathrm{DCT})$ and Sensing ( $\Phi=$ Gaussian) Matrices with total number of samples $(\mathrm{N}) \&(\mathrm{M})$

- Step6: Multiply Sparse MR Image with Basis and Sensing Matrix $\left(y=\Psi^{*} \Phi^{*} S\right)$

- Step7: After the proper compression apply IDCT and modified $l_{1}$ reconstruction algorithm

- Step8: Calculate the Value of PSNR, SSIM, and FSIM.

\section{$4 \quad$ Result and Analysis}

\subsection{Peak Signal to Noise Ratio (PSNR)}

PSNR is the quality index parameter that represents the quality of the MRI signal. It is calculated using the ratio of original MRI components to the reconstructed MRI components. The higher value of PSNR shows that the reconstructed MR image is very much close to the original image.

$$
P S N R=\frac{10 \times \log _{10}\left(\sum_{m}\left(K_{i}^{2}\right)\right)}{\sum_{m}\left[K_{i}(m)-L_{o}(m)\right]^{2}}
$$

$\mathrm{K}_{\mathrm{i}}=$ Test MR image components, $\mathrm{L}_{\mathrm{o}}=$ Reconstructed MR image components, $\mathrm{m}=$ Number of Components present in MR image.

\subsection{Mean Square Error (MSE)}

MSE is the second parameter for quality evaluation of MR image. The use of MSE is to analyze the difference between the actual MR image and its recovered version after compression. The main objective of MSE is to find out the distortion level between the actual and reconstructed signal. The relation for MSE between actual \& reconstructed signal is given in equation 7.

$$
\operatorname{MSE}(K, L)=\frac{1}{n} \sum_{1}^{n}\left(\left(K_{i}-L_{i}\right)^{2}\right)
$$

Here $\mathrm{K}=$ Original Input signal, $\mathrm{L}=$ Recovered signal, $\mathrm{n}=$ Total number of observations. 


\subsection{Compression Ratio (C/R)}

The Compression Ratio is a defined parameter that indicates how the sample components are considered in the reconstruction process out of the total number of components present in that signal or MR-image.

$$
\text { CompressionRatio }=\frac{m}{n}
$$

$\mathrm{m}=$ Samples used for reconstruction purpose of MR Image, $\mathrm{n}=$ Actual number of samples in MR Image.

\subsection{Structural Similarity Index (SSIM)}

The SSIM index is a process to find out the similarity between two images. It can be considered as a quality measurement for one image compare to another image kept the quality of the image in the main perspective. We can say it is the modified structure of the universal image quality index which was incorporated earlier [18].

$$
\operatorname{SSIM}(x, y)=\frac{\left(2 \mu_{x} \mu_{y}+c_{1}\right)\left(2 \sigma_{x y}+c_{2}\right)}{\left(\mu_{x}^{2}+\mu_{y}^{2}+c_{1}\right)\left(\sigma_{x}^{2}+\sigma_{y}^{2}+c_{2}\right)}
$$

As we have to apply the compressive sensing process on the MRI signals so for that purpose, we have taken five real-time images of the MRI data. These images are Abdomen, Brain, Feet, Thigh, and Thorax Images dimensions 64 × 64 greyscale images. We have considered three types of classification of MRI images which are mentioned earlier in this paper are $\mathrm{T} 1, \mathrm{~T} 2$, and proton density images.

Table 1. Table for $\mathrm{C} / \mathrm{R}$ v/s PSNR Values for Five MRI Signals which have T1 Relaxation

\begin{tabular}{|c|c|c|c|c|c|}
\hline \multirow{2}{*}{$(\mathbf{C} / \mathbf{R})$} & \multicolumn{5}{|c|}{ PSNR Values (For T1 Weighted Images) } \\
\cline { 2 - 6 } & Brain & Abdomen & Feet & Thigh & Thorax \\
\hline 0.12 & 18.93838 & 19.36861 & 18.18195 & 17.71327 & 21.15749 \\
\hline 0.24 & 20.32413 & 20.69414 & 20.00618 & 19.73521 & 22.84824 \\
\hline 0.36 & 22.06894 & 22.15039 & 21.59694 & 21.97017 & 24.47555 \\
\hline 0.48 & 23.58198 & 23.62765 & 23.28673 & 24.02646 & 26.17561 \\
\hline 0.61 & 25.37786 & 25.55946 & 25.05409 & 26.24926 & 27.80836 \\
\hline 0.73 & 27.22460 & 27.46423 & 27.53953 & 28.97154 & 29.43836 \\
\hline 0.85 & 30.12220 & 30.35731 & 30.91515 & 32.41923 & 32.53284 \\
\hline
\end{tabular}

The table 1 shows the Peak Signal to Noise Ratio value on varying compression ratios. The observations are carried out for MRI images based on the T1 relaxation process. Here we can identify that the value of PSNR going upward when we increase the value of the compression ratio (which means some measurements from the total number of measurements). 
Table 2. Table for $\mathrm{C} / \mathrm{R}$ v/s PSNR Values for Five MRI Signals which have T2 Relaxation

\begin{tabular}{|c|c|c|c|c|c|}
\hline \multirow{2}{*}{$(\mathbf{C} / \mathbf{R})$} & \multicolumn{5}{|c|}{ PSNR Values (For T2 Weighted Images) } \\
\cline { 2 - 6 } & Brain & Abdomen & Feet & Thigh & Thorax \\
\hline 0.12 & 19.48035 & 23.97156 & 23.51279 & 23.5314 & 24.18082 \\
\hline 0.24 & 21.16565 & 25.54832 & 25.47934 & 25.85963 & 26.39162 \\
\hline 0.36 & 22.17539 & 26.65476 & 26.59461 & 27.61357 & 27.66458 \\
\hline 0.48 & 23.69422 & 28.00451 & 28.84284 & 29.61601 & 29.28784 \\
\hline 0.61 & 25.09899 & 29.60434 & 30.71937 & 31.70183 & 31.09007 \\
\hline 0.73 & 27.22410 & 31.75730 & 32.73550 & 34.45007 & 32.55943 \\
\hline 0.85 & 29.96454 & 34.29955 & 36.20008 & 37.83451 & 35.85043 \\
\hline
\end{tabular}

Table 3. Table For C/R v/s PSNR Values for Five MRI Signals which have PD Relaxation

\begin{tabular}{|c|c|c|c|c|c|}
\hline \multirow{2}{*}{$(\mathbf{C} / \mathbf{R})$} & \multicolumn{5}{|c|}{ PSNR Values (For Proton Density Weighted Images) } \\
\cline { 2 - 6 } & Brain & Abdomen & Feet & Thigh & Thorax \\
\hline 0.12 & 13.96725 & 18.43211 & 16.65304 & 16.27933 & 18.84121 \\
\hline 0.24 & 16.13658 & 19.59346 & 17.94980 & 18.0461 & 21.20613 \\
\hline 0.36 & 17.79003 & 20.83894 & 20.11533 & 20.6163 & 22.33236 \\
\hline 0.48 & 18.94177 & 22.37726 & 21.86968 & 22.86919 & 24.00553 \\
\hline 0.61 & 20.90123 & 24.04118 & 23.87395 & 25.23256 & 25.55529 \\
\hline 0.73 & 23.39412 & 26.27313 & 25.94542 & 27.82018 & 27.67386 \\
\hline 0.85 & 26.09695 & 28.88661 & 29.44509 & 30.35384 & 30.50498 \\
\hline
\end{tabular}

The compression ratio v/s PSNR values variation is given in the table 2. Except for brain image, the value of PSNR for T2 relaxation is much higher than the T1 relaxation process. We can easily find by the table 3 that the Proton Density MRI image after the reconstruction has lower PSNR values than the $\mathrm{T} 1$ and $\mathrm{T} 2$ relaxation.

Table 4. Table For C/R v/s SSIM Values for Five MRI Signals which have T1 Relaxation

\begin{tabular}{|c|c|c|c|c|c|}
\hline \multirow{2}{*}{$(\mathbf{C} / \mathbf{R})$} & \multicolumn{5}{|c|}{ SSIM Values (For T1 weighted Images) } \\
\cline { 2 - 6 } & Brain & Abdomen & Feet & Thigh & Thorax \\
\hline 0.12 & 0.21898 & 0.23194 & 0.15865 & 0.19912 & 0.295578 \\
\hline 0.24 & 0.28685 & 0.33501 & 0.26492 & 0.29315 & 0.401657 \\
\hline 0.36 & 0.38312 & 0.43101 & 0.34520 & 0.40479 & 0.503201 \\
\hline 0.48 & 0.43948 & 0.52986 & 0.39979 & 0.49139 & 0.597597 \\
\hline 0.61 & 0.52334 & 0.62558 & 0.46285 & 0.59052 & 0.675046 \\
\hline 0.73 & 0.59066 & 0.71758 & 0.51707 & 0.67837 & 0.741893 \\
\hline 0.85 & 0.69260 & 0.81597 & 0.63179 & 0.79260 & 0.834324 \\
\hline
\end{tabular}


Table 5. Table for $\mathrm{C} / \mathrm{R}$ v/s SSIM Values for Five MRI Signals which have T2 Relaxation

\begin{tabular}{|c|c|c|c|c|c|}
\hline \multirow{2}{*}{$(\mathbf{C} / \mathbf{R})$} & \multicolumn{5}{|c|}{ SSIM Values (For T2 Weighted Images) } \\
\cline { 2 - 6 } & Brain & Abdomen & Feet & Thigh & Thorax \\
\hline 0.12 & 0.28865 & 0.37844 & 0.35723 & 0.35257 & 0.38512 \\
\hline 0.24 & 0.34167 & 0.46097 & 0.47988 & 0.47082 & 0.53597 \\
\hline 0.36 & 0.37338 & 0.53927 & 0.49485 & 0.54865 & 0.60269 \\
\hline 0.48 & 0.48187 & 0.62506 & 0.59226 & 0.65809 & 0.68187 \\
\hline 0.61 & 0.53215 & 0.70495 & 0.65653 & 0.73290 & 0.75193 \\
\hline 0.73 & 0.60963 & 0.79099 & 0.71711 & 0.82102 & 0.81067 \\
\hline 0.85 & 0.69366 & 0.87211 & 0.81566 & 0.89266 & 0.89208 \\
\hline
\end{tabular}

Table 6. Table for C/R v/s SSIM Values for Five MRI Signals which have PD Relaxation

\begin{tabular}{|c|c|c|c|c|c|}
\hline \multirow{2}{*}{$(\mathbf{C} / \mathbf{R})$} & \multicolumn{5}{|c|}{ SSIM Values (For Proton Density Weighted Images) } \\
\cline { 2 - 6 } & Brain & Abdomen & Feet & Thigh & Thorax \\
\hline 0.12 & 0.14578 & 0.22028 & 0.21729 & 0.19123 & 0.23435 \\
\hline 0.24 & 0.24310 & 0.32341 & 0.25173 & 0.27133 & 0.38378 \\
\hline 0.36 & 0.31649 & 0.40698 & 0.34685 & 0.40560 & 0.45988 \\
\hline 0.48 & 0.37375 & 0.51684 & 0.40262 & 0.49348 & 0.56575 \\
\hline 0.61 & 0.45396 & 0.60451 & 0.46866 & 0.59094 & 0.63403 \\
\hline 0.73 & 0.52570 & 0.70775 & 0.51651 & 0.68878 & 0.72311 \\
\hline 0.85 & 0.61998 & 0.80073 & 0.63012 & 0.79612 & 0.81584 \\
\hline
\end{tabular}

Tables 4, 5, and 6 which are shown above indicates the clear picture related to the compression ratio and the structural similarity index (SSIM) value. Here we found that the SSIM value for T2 relaxation is much higher than the values for T1 and Proton density MRI images.

In the next part of this analysis, we are going to represents the reconstructed Images after the compressive sensing applied to the original test images. Here we have considered the minimum compression ratio that is about 500 measurement samples from 4096 total number of samples and maximum compression ratio that is 3500 samples out of 4096 samples. The table comprises of MRI signal Name and type with the exact reconstruction image.

Table 7. Original v/s Reconstructed Images For Different Weighted MRI Images

\begin{tabular}{|c|c|c|}
\hline $\begin{array}{l}\text { MRI Image Name } \\
\text { and Type }\end{array}$ & For Least Compression Ratio (500/4096) & $\begin{array}{l}\text { For Maximum Compression Ratio } \\
\qquad(3500 / 4096)\end{array}$ \\
\hline \multicolumn{3}{|c|}{$B R A I N$} \\
\hline \multirow[t]{2}{*}{ T-1 Weighted } & & \\
\hline & 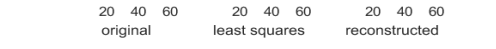 & 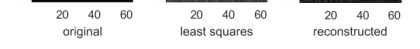 \\
\hline T-2 Weighted & & \\
\hline & 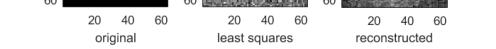 & 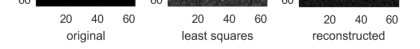 \\
\hline
\end{tabular}




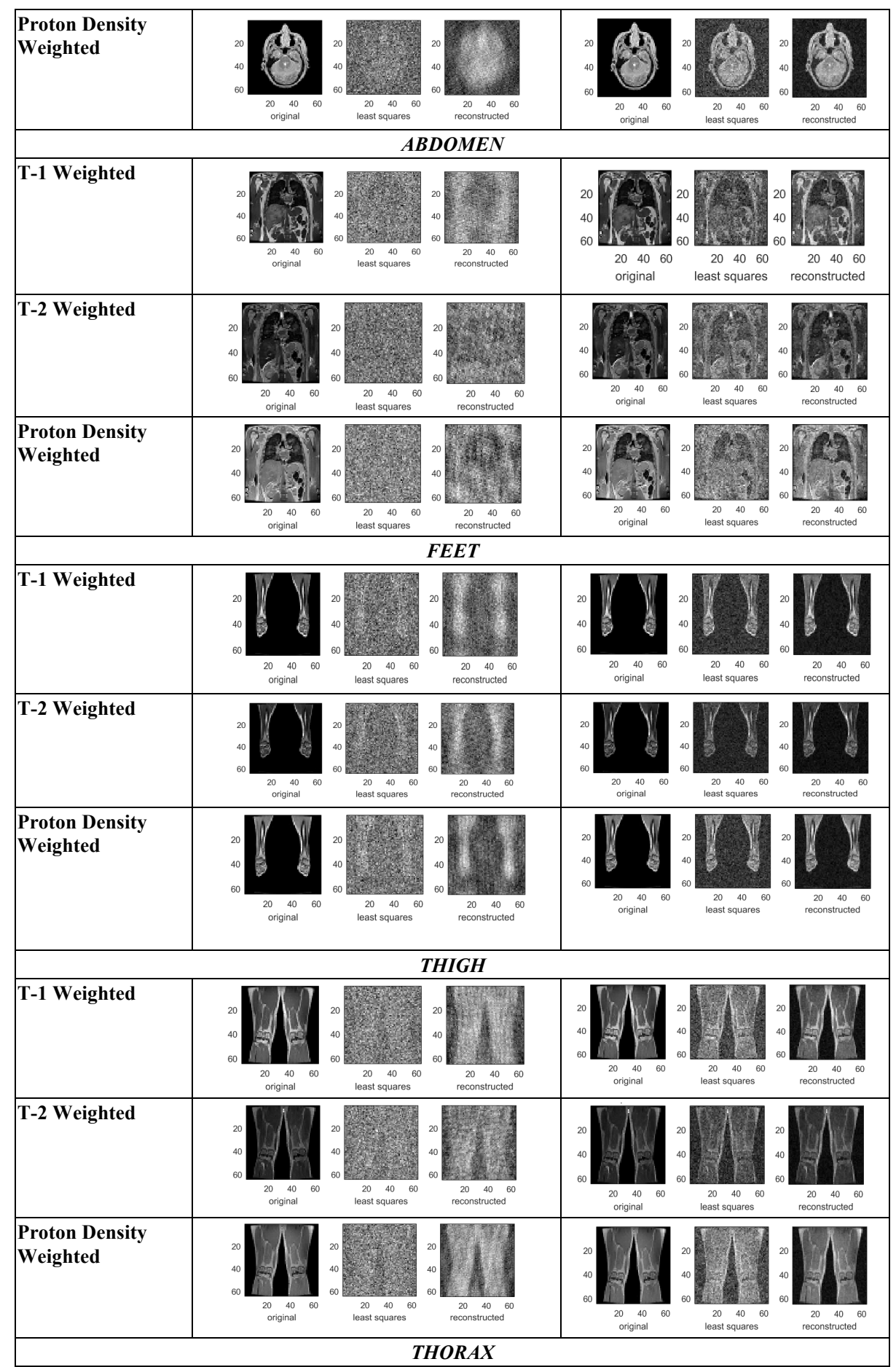




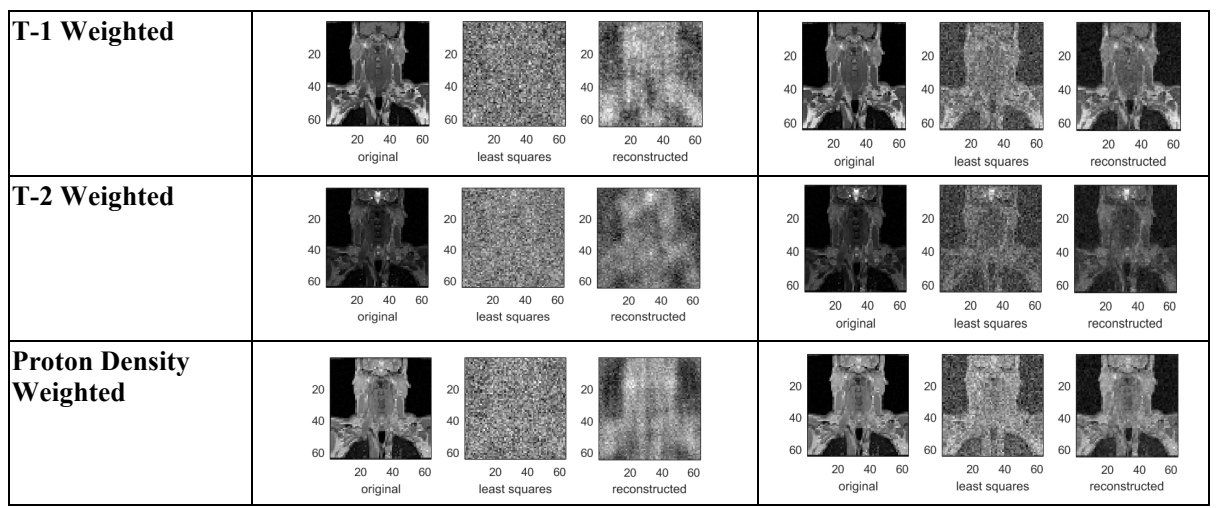

In the next segment of the observation, we are going to represent the Compression Ratio v/s Mean square curves for the different kinds of relaxation strategies with five types of MRI images that are for Brain, Abdomen, Feet, Thigh, and Thorax. MSE values for the Proton Density Images are much higher than the T1 and T2 values. That indicates that the proton density relaxation process has not much clarity in the images after the compression and recovery.

Table 8. Table For Compression Ratio v/s MSE value curves for Different Types of MRI Images

\begin{tabular}{|l|c|c|c|}
\hline Name of MRI Image & Compression Ratio v/s MSE Curve for T1, T2, and Proton Density \\
Weighted Image
\end{tabular}




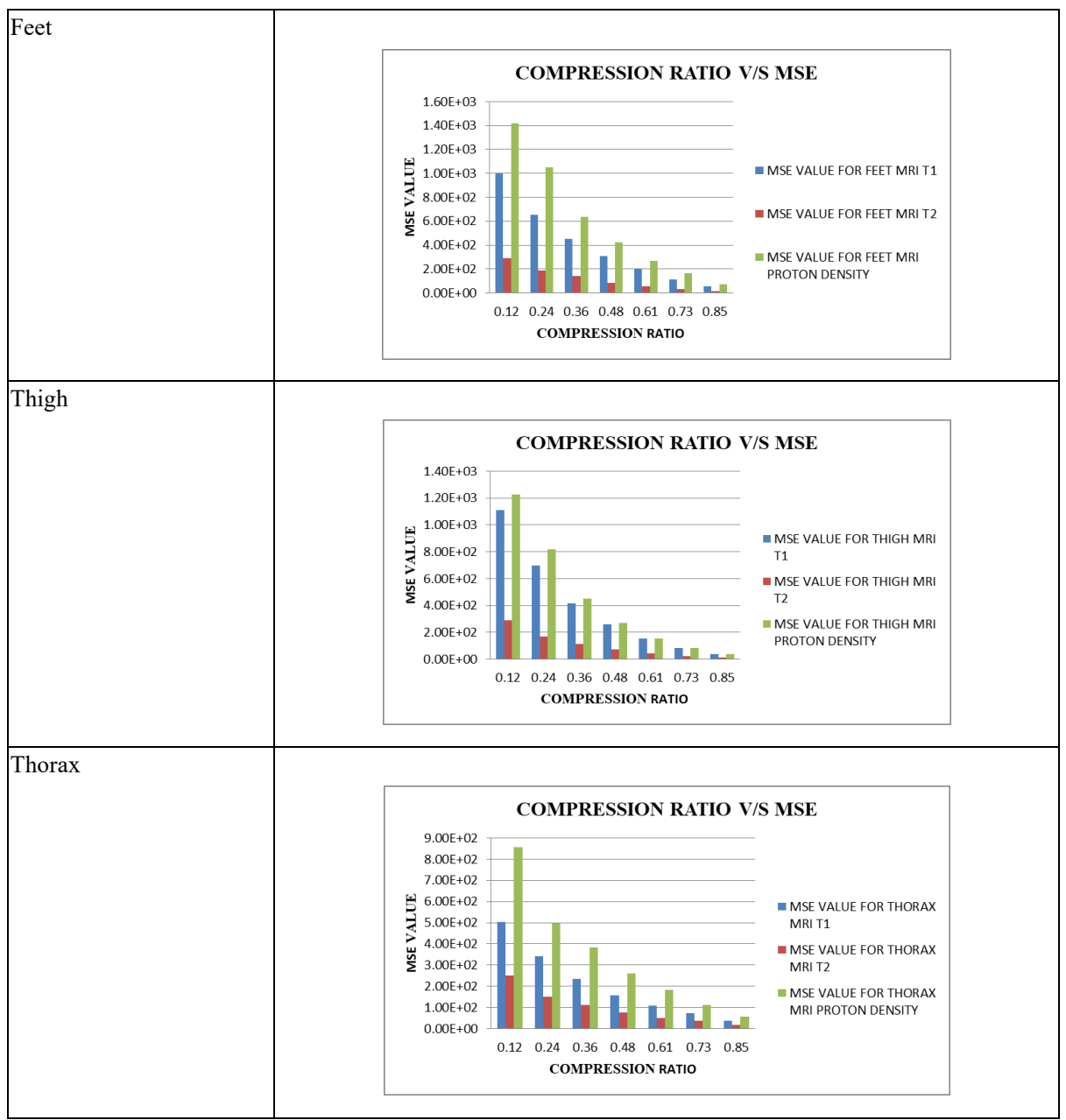

In this section, we discuss various observations that are done under the compression and reconstruction process. We have discussed three parameters that show the quality of a reconstructed image, these parameters are PSNR, MSE, and SSIM. As we know that different images have different information in the given frame. So, it is very typical to find out a unique difference between the images having the same dimension (64x64 in this case). The next section is the conclusion section which is going to elaborate on various findings in this paper.

\section{Conclusion}

In this overall experiment, our prime objective is to find out how the different relaxation strategies and the MRI signals behave after applying the compressive sensing theory. We have several findings in our experimental research. Now we are going to 
mention the conclusions which we obtain after the experiments. The overall analysis is based on the application of a compressive sensing process on the MRI image signal. Some of the key points which are the essence of this analysis are as follows.

- Based on the comparison we can easily predict that the reconstruction of the T2 weighted image is much favourable than T1 and Proton Density weighted images and can provide a high value of quality parameters by using few samples. The higher values of PSNR, MSE, and SSIM prove this fact.

- The second most important fact is that after the $50 \%$ value of compression the Peak signal to noise ratio varies very abruptly.

- The mean square error value shows how much reconstruction is done perfectly or we can say that how much error in the reconstructed image concerning the original image is very high in the Proton Density MRI images. This means that this type of image after the reconstruction provides the least efficient image reconstruction.

- Image reconstruction is highly dependent on the structure of the MRI image.

- The reconstruction algorithm which is used is much effective than that of other algorithms used for similar kinds of images.

- By investigating the reconstructed images, we can say that the algorithm which is used is efficient enough to provide a good level of reconstruction which is shown by SSIM values.

\section{Acknowledgement}

This research was "Supported by Visvesvaraya Ph.D. Scheme, MeitY, Govt. of India with unique awardee number "MEITY-PHD-2946".

\section{$7 \quad$ References}

[1] Ridgway, John P. "Cardiovascular magnetic resonance physics for clinicians: part I." Journal of cardiovascular magnetic resonance 12, no. 1 (2010): 71. https://doi.org/10.1186/ $1532-429 \mathrm{x}-12-71$

[2] Donoho, D., “Compressed sensing," IEEE Trans. Inform. Theory, vol. 52, no. 4, pp. 12891306, Apr. 2006. https://doi.org/10.1109/tit.2006.871582

[3] R. Baraniuk. Compressive sensing. IEEE Signal Processing Mag., 24(4):118-120, 124, 2007.

[4] Weiss, L., \& McDonough, R. N. (1963). Prony's method, Z-transforms, and Padé approximation. Siam Review, 5(2), 145-149. https://doi.org/10.1137/1005035

[5] V. (1980). Another generalization of Carathéodory's theorem. Archiv der Mathematik, 34(1), 560-562.

[6] Goluzina, E. G. E. (1985). Ranges of values of systems of functionals in certain classes of regular functions. Mathematical Notes, 37(6), 438-442. https://doi.org/10.1007/bf01157 $\underline{679}$

[7] K. Gedalyahu and Y. C. Eldar. Time-delay estimation from low-rate samples: A union of subspaces approach. IEEE Trans. Signal Processing, 58(6):3017-3031, 2010. 
[8] K. Gedalyahu, R. Tur, and Y. C. Eldar. Multichannel sampling of pulse streams at the rate of innovation. To appear in IEEE Trans. Signal Processing, 2011. https://doi.org/10. $\underline{1109 / \mathrm{sam} .2010 .5606714}$

[9] M. Mishali and Y. C. Eldar. From theory to practice: Sub-Nyquist sampling of sparse wideband analog signals. IEEE J. Select. Top. Signal Processing, 4(2):375-391, 2010. https://doi.org/10.1109/jstsp.2010.2042414

[10] R. Robucci, L. Chiu, J. Gray, J. Romberg, P. Hasler, and D. Anderson. Compressive sensing on a CMOS separable transform image sensor. In Proc. IEEE Int. Conf. Acoust, Speech, and Signal Processing (ICASSP), Las Vegas, NV, Apr. 2008. https://doi.org/ 10.1109/icassp.2008.4518812

[11] Shukla, U. P., Patel, N. B., \& Joshi, A. M., "A survey on recent advances in speech compressive sensing," 2013 IEEE International Multi-Conference on Automation, Computing, Communication, Control and Compressed Sensing (iMac4s), pp. 276-280, March-2013. https://doi.org/10.1109/imac4s.2013.6526422

[12] Mardani, Morteza, Enhao Gong, Joseph Y. Cheng, Shreyas S. Vasanawala, Greg Zaharchuk, Lei Xing, and John M. Pauly. "Deep generative adversarial neural networks for compressive sensing MRI." IEEE transactions on medical imaging 38, no. 1 (2018): 167-179. https://doi.org/10.1109/tmi.2018.2858752

[13] Yuan, Lianjun, Yunyi Li, Fei Dai, Yan Long, Xiefeng Cheng, and Guan Gui. "Analysis \$ $\left\{\left\{L_{-}\{\{1 / 2\}\}\right\}\right\}$ \$ Regularization: Iterative Half Thresholding Algorithm for CS-MRI." IEEE Access 7 (2019): 79366-79373. https://doi.org/10.1109/access.2019.2923171

[14] Gözcü, Baran, Rabeeh Karimi Mahabadi, Yen-Huan Li, Efe Ilıcak, Tolga Cukur, Jonathan Scarlett, and Volkan Cevher. "Learning-based compressive MRI." IEEE transactions on medical imaging 37, no. 6 (2018): 1394-1406. https://doi.org/10.1109/tmi.2018.2832540

[15] Xi, Yan, Jun Zhao, James R. Bennett, Mitchel R. Stacy, Albert J. Sinusas, and Ge Wang. "Simultaneous CT-MRI reconstruction for constrained imaging geometries using structural coupling and compressive sensing." IEEE Transactions on Biomedical Engineering 63, no. 6 (2015): 1301-1309. https://doi.org/10.1109/tbme.2015.2487779

[16] Saucedo, Andres, Stamatios Lefkimmiatis, Novena Rangwala, and Kyunghyun Sung. "Improved computational efficiency of locally low-rank MRI reconstruction using iterative random patch adjustments." IEEE transactions on medical imaging 36, no. 6 (2017): 1209-1220. https://doi.org/10.1109/tmi.2017.2659742

[17] Tashan, Tariq, and Maher Al-Azawi. "Multilevel magnetic resonance imaging compression using compressive sensing." IET Image Processing 12, no. 12 (2018): 2186-2191. https://doi.org/10.1049/iet-ipr.2018.5611

[18] Wang, Zhou, Alan C. Bovik, Hamid R. Sheikh, and Eero P. Simoncelli. "Image quality assessment: from error visibility to structural similarity." IEEE transactions on image processing 13, no. 4 (2004): 600-612. https://doi.org/10.1109/tip.2003.819861

[19] Saudagar, Abdul. "Biomedical Image Compression Techniques for Clinical Image Processing." (2020): 133-154.

[20] Lalitha, Y. Sri, Katapally Manognya, Pabba Keerthana, and Mudunuri Vineetha. "Efficient Tumor Detection in MRI Brain Images." iJOE 16, no. 13 (2020): 123. https://doi.org/10. 3991/ijoe.v16i13.18613

[21] Kalyani, Chenigaram, Kama Ramudu, and Ganta Raghotham Reddy. "Enhancement and Segmentation of Medical Images Using AGCWD and ORACM." International Journal of Online \& Biomedical Engineering 16, no. 13 (2020). https://doi.org/10.3991/ijoe. $\underline{\mathrm{v} 16 \mathrm{i} 13.18501}$ 


\section{Authors}

Vivek Upadhyaya graduated with his "Bachelor of Engineering" in Electronics and Communication Engineering from Rajasthan University, Rajasthan, India in 2009. He has done M. Tech in Electronics and Communication from Malaviya National Institute of Technology, Jaipur, in the year 2016 and a Ph.D. (Pursuing) from Malaviya National Institute of technology. He has been the recipient of the Visvesvaraya scholarship given by the Ministry of Electronics \& I.T. (Govt. of India) during his Ph.D. His research work is focused on Medical Imaging and Data Compression. 2018rec9028@mnit.ac.in

Prof. Mohammad Salim has more than 35 years of teaching experience and presently serving as Professor in the Department of Electronics and Communication, Malaviya National Institute of Technology, Jaipur, India. He has guided more than 50 M. Tech. thesis, 45 undergraduate projects, and $5 \mathrm{PhD}$ thesis. He has been to Sheffield Hallam University, Sheffield, the U.K. for eight months under the U.K.-India REC project. Email id: (msalim.ece@mnit.ac.in)

Article submitted 2020-12-25. Resubmitted 2021-02-23. Final acceptance 2021-02-24. Final version published as submitted by the authors. 